\title{
Development and characterization of 70 novel microsatellite markers for the sea cucumber (Apostichopus japonicus)
}

\author{
W. Peng, Z.M. Bao, H.X. Du, J.J. Yan, L.L. Zhang and J.J. Hu \\ Key Laboratory of Marine Genetics and Breeding, \\ College of Marine Life Sciences, Ocean University of China, \\ Qingdao, P.R. China \\ Corresponding author: L.L. Zhang \\ E-mail: lingling80@ouc.edu.cn
}

Genet. Mol. Res. 11 (1): 434-439 (2012)

Received March 18, 2011

Accepted December 2, 2011

Published February 24, 2012

DOI http://dx.doi.org/10.4238/2012.February.24.2

\begin{abstract}
The sea cucumber (Apostichopus japonicus) is an important item in Asian cuisine. It is currently produced through aquaculture, especially in China, after being overexploited in the wild in the 1990s. We isolated 70 novel polymorphic microsatellite loci using an enrichment-colony hybridization protocol. All loci were characterized in 48 individuals from a natural population in Rongcheng (Shandong, China) using genomic DNA isolated from muscle tissue. The number of alleles ranged from 2 to 17 (mean 7.0), and the observed and expected heterozygosities varied from 0.0010 to 1.0000 and from 0.2125 to 0.9477 , respectively. Thirty-one of the 70 loci exhibited departure from Hardy-Weinberg equilibrium. These microsatellite markers should be useful resources for population genetic studies and
\end{abstract}


for molecular marker-assisted breeding of A. japonicus.

Key words: Apostichopus japonicus; Microsatellite; Polymorphic marker

\section{INTRODUCTION}

The sea cucumber, Apostichopus japonicus, is naturally distributed along the coast of China, Japan and Korea in the Western Pacific Ocean (Chen, 1990). This species is considered a delicacy in these countries. Due to strong consumer preferences, the demand for sea cucumber products increases insatiably. However, large demand has resulted in overexploitation and environmental deterioration. To satisfy the increasing demand and protect marine resources, the sea cucumber industry has developed rapidly in recent years. In an effort to increase the efficiency and profitability of aquaculture production systems, genome analysis on the sea cucumber has been carried out. To conduct genetic studies, molecular markers such as polymorphic microsatellites have been widely used as a principal tool. Although some microsatellite loci have been developed for A. japonicus (Kanno et al., 2005; Chen and Li, 2007; Zhan et al., 2007; Peng et al., 2009), more microsatellite loci are still needed to promote the implementation of genetic analysis and breeding programs. In this paper, we report the isolation and characterization of 70 novel microsatellite loci in A. japonicus.

\section{MATERIAL AND METHODS}

\section{DNA extraction and enrichment for microsatellites}

Genomic DNA was extracted from muscle tissue according to the protocol described by Zhan et al. (2007). Microsatellite markers were isolated by the enrichmentcolony hybridization method reported by Fischer and Bachmann (1998) and Zhan et al. (2007) with some modifications. Approximately $10 \mu \mathrm{g}$ extracted DNA was digested with $50 \mathrm{U} \mathrm{AluI}$ restriction enzyme at $37^{\circ} \mathrm{C}$ for $6 \mathrm{~h}$. The 400 - to 1500 -bp DNA fragments were selected with a $1 \%$ low melting temperature agarose gel. The 21 -mer (5'-CTCTTGCTTGA ATTCGGACTA-3') and phosphorylated 25-mer (5'-pTAGTCCGAATTCAAGCAAGAG CACA-3') adaptors were ligated to the selected fragments using $\mathrm{T}_{4}$ DNA ligase at $16^{\circ} \mathrm{C}$ for $12 \mathrm{~h}$. The ligated fragments were amplified using a single adaptor (21-mer) as primers. The PCRs were performed as follows: $5 \mathrm{~min}$ at $94^{\circ} \mathrm{C}$, and then 25 cycles of denaturation for $45 \mathrm{~s}$ at $94^{\circ} \mathrm{C}$, annealing for $45 \mathrm{~s}$ at $55^{\circ} \mathrm{C}$, and extension for $45 \mathrm{~s}$ at $72^{\circ} \mathrm{C}$, and a final extension for $10 \mathrm{~min}$ at $72^{\circ} \mathrm{C}$. The amplified products were enriched for microsatellite sequences through hybridization with $(\mathrm{AC})_{15},(\mathrm{AG})_{15}$ or $(\mathrm{ACAG})_{8}$ oligonucleotides bound to nylon membranes. All hybridizations took place at $37^{\circ} \mathrm{C}$ for $12 \mathrm{~h}$ followed by washes of $58^{\circ} \mathrm{C}$ in $2 \mathrm{X} \mathrm{SSC}, 1 \% \mathrm{SDS}$ for three times. The quantity of captured DNA was increased by reamplification with 21-mer primer and rTaq DNA polymerase (denaturation for $5 \mathrm{~min}$ at $94^{\circ} \mathrm{C} ; 25$ cycles of $45 \mathrm{~s}$ at $94^{\circ} \mathrm{C}, 45 \mathrm{~s}$ at $55^{\circ} \mathrm{C}$, and extension for $45 \mathrm{~s}$ at $72^{\circ} \mathrm{C}$, and an additional extension for $1 \mathrm{~h}$ at $72^{\circ} \mathrm{C}$ ). The PCR products were cloned into pMD 18-T vector (TaKaRa) and transformed into competent Escherichia coli DH5 $\alpha$ cells. Then, the clones were rearranged in order on a new agar plate and transferred onto nylon membranes. The 
blots were screened with digoxigenin labeled $(\mathrm{AC})_{15},(\mathrm{AG})_{15}$ and $(\mathrm{ACAG})_{8}$ probes (Roche Applied Science), then, positive signals were generated using the DIG detection system (Roche Applied Science).

\section{Primer design and PCR amplification}

Some of the positive clones, which were screened via PCR and 21-mer primer, were sequenced using the sequencing primer M13-47. After discarding redundant sequences and examining the length and GC content of the flanking regions, primers were designed using the primer premier 5 software (Premier Biosoft International). After optimization of the PCR parameter, primer pairs that produced clear amplifications of the expected size were selected for microsatellite polymorphism analysis. A total of 48 sea cucumber individuals from Rongcheng (Shandong, China) were sampled to test for polymorphisms. PCR amplifications were carried out in a $20-\mu \mathrm{L}$ reaction mixture containing $1 \mathrm{U}$ rTaq DNA polymerase (TaKaRa), 1X PCR buffer, $1.5 \mathrm{mM} \mathrm{MgCl} 2,0.2 \mathrm{mM}$ dNTP mix, $0.2 \mu \mathrm{M}$ of each primer set, and 50-100 ng template DNA. PCR cycles were as follows: initial denaturation of $94^{\circ} \mathrm{C}$ for $5 \mathrm{~min}$, followed by 35 cycles of $94^{\circ} \mathrm{C}$ for $30 \mathrm{~s}$, primer specific annealing temperature for $30 \mathrm{~s}, 72^{\circ} \mathrm{C}$ for $30 \mathrm{~s}$, and a final extension step at $72^{\circ} \mathrm{C}$ for $5 \mathrm{~min}$. PCRs were performed in a thermal cycler (GeneAmp PCR System 9700, Perkin-Elmer ABI). The amplification products were separated by electrophoresis on a $12 \%$ nondenaturing polyacrylamide gel, stained with ethiduim bromide and visualized under UV light. Allele sizes were estimated using a 100-bp ladder molecular size standard (Invitrogen).

\section{Data analysis}

POPGENE32 (Yeh and Boyle, 1997) and ARLEQUIN softwares (Schneider et al., $2000)$ were used to calculate the number of alleles, observed $\left(H_{\mathrm{O}}\right)$ and expected $\left(H_{\mathrm{E}}\right)$ heterozygosities, Hardy-Weinberg equilibrium (HWE) and linkage disequilibrium, respectively. All results for multiple tests were corrected using Bonferroni's correction (Rice, 1989).

\section{RESULTS AND DISCUSSION}

Of the 1000 recombinant colonies screened, 723 gave a positive signal $(72.3 \%)$. Plasmids from 300 positive clones randomly selected were sequenced, 295 contained at least one microsatellite (98.3\%). After discarding redundant sequences and examining the length and GC content of the flanking regions, primers were designed for 121 sequences.

Of the 121 primer pairs, 70 loci showed clear and scorable amplification patterns, and were polymorphic in the 48 sea cucumber individuals tested. The number of alleles observed per polymorphic locus varied from 2 to 17 (mean 7.0). The $H_{\mathrm{O}}$ varied from 0.0010 to 1.0000 , while $H_{\mathrm{E}}$ ranged from 0.2125 to 0.9477 . Significant deviation from HWE $(\mathrm{P}<0.05)$ was detected at 31 loci, which showed a significant heterozygote deficiency (Table 1). The presence of null alleles or population structure may be responsible for the deviations from HWE. Eight locus pairs displayed linkage disequilibrium after Bonferroni's correction. These polymorphic microsatellite markers developed in the present study will provide a useful tool for further genetic studies of $A$. japonicus. 
Table 1. Characterization of 70 novel microsatellite markers for Apostichopus japonicas.

\begin{tabular}{|c|c|c|c|c|c|c|c|}
\hline Locus & Primer sequence $\left(5^{\prime}-3^{\prime}\right)$ & $T_{\mathrm{a}}\left({ }^{\circ} \mathrm{C}\right)$ & Repeat motifs & Range & $N_{\mathrm{A}}$ & $H_{\mathrm{O}} / H_{\mathrm{E}}$ & Accession No \\
\hline $1 \mathrm{~S} 45$ & $\begin{array}{l}\text { F: CAGTGATGACATTATATTGGGC } \\
\text { R: TAGGAAGCGTCCTGTAGTTGC }\end{array}$ & 56 & $(\mathrm{TG})_{11}$ & $157-183$ & 9 & $\begin{array}{l}0.2917 \\
0.8262 *\end{array}$ & JF692822 \\
\hline $1 \mathrm{~S} 46$ & $\begin{array}{l}\text { F: TTGAAGTACATACACTTTGCC } \\
\text { R: ATTACTCCTAAATTGAGTCCC }\end{array}$ & 50 & $(\mathrm{CA})_{31}$ & $120-158$ & 9 & $\begin{array}{l}0.6429 \\
0.8889\end{array}$ & JF692823 \\
\hline $1 \mathrm{~S} 55$ & $\begin{array}{l}\text { F: CATGCTATGATAAGTCCTCCTG } \\
\text { R: AAGATGAATGCCAATTCCCG }\end{array}$ & 56 & $(\mathrm{TG})_{8}$ & 254-308 & 6 & $\begin{array}{l}0.5455 \\
0.8425^{*}\end{array}$ & JF692826 \\
\hline $3 \mathrm{~S} 08$ & $\begin{array}{l}\text { F: TTGAGTACACAAAGCAAGCG } \\
\text { R: GGACTAAAATGGTGAGTAGGAC }\end{array}$ & 45 & $(\mathrm{CA})_{14}$ & $129-161$ & 10 & $\begin{array}{l}0.4167 \\
0.8892^{*}\end{array}$ & JF692848 \\
\hline $3 \mathrm{~S} 14$ & $\begin{array}{l}\text { F: GGAAACCATCATGTTAATGC } \\
\text { R: ATCAACACTGCCAATTTGTG }\end{array}$ & 58 & $(\mathrm{TC})_{7}$ & $231-265$ & 7 & $\begin{array}{l}0.1739 \\
0.7072 *\end{array}$ & JF692851 \\
\hline $3 \mathrm{~S} 16$ & $\begin{array}{l}\text { F: TTATCCTCATCCATACCGTC } \\
\text { R: AAGTTATCTGTTCACCGTCG }\end{array}$ & 58 & $(\mathrm{AG})_{10} \mathrm{GTG}(\mathrm{GA})_{22}$ & $255-291$ & 5 & $\begin{array}{l}0.8261 \\
0.7923\end{array}$ & JF692852 \\
\hline $3 \mathrm{~S} 17$ & $\begin{array}{l}\text { F: CTGTGTTTGAAGAGCAAAGT } \\
\text { R: CATTCCATCTAACCCAGTATC }\end{array}$ & 45 & $(\mathrm{AG})_{26}$ & $177-203$ & 8 & $\begin{array}{l}0.8260 \\
0.8029\end{array}$ & JF692853 \\
\hline $3 \mathrm{~S} 18$ & $\begin{array}{l}\text { F: GCACTACCCACGACATATAAG } \\
\text { R: ATCAGCAACCACCAAGCAAG }\end{array}$ & 62 & $(\mathrm{AC})_{7} \ldots(\mathrm{CA})_{14}$ & $207-229$ & 3 & $\begin{array}{l}0.9583 \\
0.6658^{*}\end{array}$ & JF692854 \\
\hline $3 \mathrm{~S} 22$ & $\begin{array}{l}\text { F: GCTTATCATAGATGTCAGTTTGC } \\
\text { R: CAATCTGTGATTTCATAGGTGC }\end{array}$ & 58 & $(\mathrm{TG})_{9}$ TATGG $(\mathrm{GT})_{10}$ & $188-224$ & 8 & $\begin{array}{l}0.4348 \\
0.7101^{*}\end{array}$ & JF692856 \\
\hline $3 \mathrm{~S} 24$ & $\begin{array}{l}\text { F: CAACAGGTAAAGAATGAAATG } \\
\text { R: ATTAGCAAGTAAACGTCTGC }\end{array}$ & 62 & $(\mathrm{GT})_{8}$ & $135-167$ & 7 & $\begin{array}{l}0.2273 \\
0.8383^{*}\end{array}$ & JF692857 \\
\hline $3 \mathrm{~S} 29$ & $\begin{array}{l}\text { F: AAAGGAACCTATGCAGTCAGG } \\
\text { R: GTGGATGGTTTTGCACATTG }\end{array}$ & 62 & $(\mathrm{GT})_{7}$ & $175-211$ & 8 & $\begin{array}{l}0.4545 \\
0.8784^{*}\end{array}$ & JF692860 \\
\hline $3 \mathrm{~S} 33$ & $\begin{array}{l}\text { F: ACCAGTTAACAAGTTAAGATGGC } \\
\text { R: CATTTCGGTTACAAGCACCTC }\end{array}$ & 50 & $(\mathrm{CA})_{27}$ & $166-176$ & 3 & $\begin{array}{l}0.2777 \\
0.2125\end{array}$ & JF692861 \\
\hline $3 \mathrm{~S} 34$ & $\begin{array}{l}\text { F: CAGAATTGACGAGATACGATAAG } \\
\text { R: CAGAAATGGACCAGGAAACC }\end{array}$ & 50 & $(\mathrm{GT})_{26}$ & $233-251$ & 4 & $\begin{array}{l}0.7778 \\
0.6937\end{array}$ & JF692862 \\
\hline $4 \mathrm{~S} 02$ & $\begin{array}{l}\text { F: AAGCGTAACAGAAAGAACAC } \\
\text { R: CCTCACCAGTAACACAGAATC }\end{array}$ & 56 & $(\mathrm{CA})_{12}$ & $144-186$ & 13 & $\begin{array}{l}0.9167 \\
0.9131\end{array}$ & JF692827 \\
\hline $4 \mathrm{~S} 05$ & $\begin{array}{l}\text { F: CCAATGCTTTACTTGATACAACC } \\
\text { R: CATGCACTTTAACAGTCCTCAG }\end{array}$ & 48 & $(\mathrm{GT})_{13}(\mathrm{GA})_{29}$ & $196-226$ & 4 & $\begin{array}{l}0.2632 \\
0.3926\end{array}$ & JF692830 \\
\hline $4 \mathrm{~S} 08$ & $\begin{array}{l}\text { F: GCATGTTAGACATTATGGTTCAG } \\
\text { R: GTACCCAAGCAAGTGTCACAG }\end{array}$ & 56 & $(\mathrm{GT})_{22}$ & $180-262$ & 11 & $\begin{array}{l}0.9583 \\
0.9113^{*}\end{array}$ & JF692831 \\
\hline $4 \mathrm{~S} 23$ & $\begin{array}{l}\text { F: CCTATCCATTTTCCAAGTGTC } \\
\text { R: AAGCCCTGAAATCATGTCAAG }\end{array}$ & 56 & $(\mathrm{TG})_{11}$ & $167-191$ & 11 & $\begin{array}{l}0.5417 \\
0.8892\end{array}$ & JF692884 \\
\hline AJ04 & $\begin{array}{l}\text { F: TTACAACTCTTCTCCCCCTC } \\
\text { R: TCGTATATCCCTGTTCTCCTC }\end{array}$ & 62 & $(\mathrm{GA})_{14}$ & $232-256$ & 9 & $\begin{array}{l}0.1818 \\
0.8721^{*}\end{array}$ & JF692788 \\
\hline AJ06 & $\begin{array}{l}\text { F: TATTGTAGGAAGGGTAAGTCG } \\
\text { R: CTGAGTGTTGAATTTCTGGC }\end{array}$ & 62 & $(\mathrm{GCAC})_{4}$ & $240-252$ & 3 & $\begin{array}{l}0.0010 \\
0.5643^{*}\end{array}$ & JF692789 \\
\hline AJ08 & $\begin{array}{l}\text { F: CTACATCTTATGCAACCCTGC } \\
\text { R: GGAACTCATTGTTTTGCTTACC }\end{array}$ & 58 & $(\mathrm{CT})_{23}(\mathrm{GT})_{31}$ & $199-293$ & 8 & $\begin{array}{l}0.5238 \\
0.5761\end{array}$ & JF692791 \\
\hline AJ09 & $\begin{array}{l}\text { F: AATATGTTAGAGAAGGTCCTCC } \\
\text { R: GTAAGACAGACACATACAAAGG }\end{array}$ & 60 & $(\mathrm{CTGT})_{12}$ & $190-246$ & 12 & $\begin{array}{l}0.4706 \\
0.8930\end{array}$ & JF692792 \\
\hline AJ10 & $\begin{array}{l}\text { F: CAGAGGTTTCCTATTGTTAAGTG } \\
\text { R: CGTTCTGTTGTCTGACATTTC }\end{array}$ & 56 & $(\mathrm{AGAC})_{21}(\mathrm{AGAT})_{6}$ & $243-271$ & 3 & $\begin{array}{l}0.3750 \\
0.5667\end{array}$ & JF692793 \\
\hline $\mathrm{AJ} 13$ & $\begin{array}{l}\text { F: CCAACACTGGAGTCTAACGAG } \\
\text { R: GATAACACAGTGACAAGCAAGG }\end{array}$ & 62 & $(\mathrm{CTTCT})_{3}$ & $243-313$ & 11 & $\begin{array}{l}0.3750 \\
0.8342^{*}\end{array}$ & JF692796 \\
\hline AJ14 & $\begin{array}{l}\text { F: GATAGACGGATAGATGGATAG } \\
\text { R: CGATACAGTGTTACAAACAAGC }\end{array}$ & 62 & $(\mathrm{GACA})_{12}$ & $302-362$ & 11 & $\begin{array}{l}0.6667 \\
0.9111\end{array}$ & JF692797 \\
\hline AJ17 & $\begin{array}{l}\text { F: CAAAGTGCTCTATTTCCCTCC } \\
\text { R: TTCCTGCTTTTCAAACCACC }\end{array}$ & 60 & $(\mathrm{CTGT})_{12}$ & $122-206$ & 17 & $\begin{array}{l}0.5417 \\
0.9477\end{array}$ & JF692798 \\
\hline AJ19 & $\begin{array}{l}\text { F: TGCTCCTTCTGTTTCTACCTTC } \\
\text { R: GGCTCCCCCATTTTTATCAC }\end{array}$ & 60 & $(\mathrm{CAGA})_{14}$ & $328-384$ & 10 & $\begin{array}{l}0.5417 \\
0.8537\end{array}$ & JF692799 \\
\hline AJ28 & $\begin{array}{l}\text { F: GATGATTCTGATACACATACCC } \\
\text { R: CATCTGAAGTCAAAGCCGAG }\end{array}$ & 60 & $(\mathrm{AGAC})_{11}(\mathrm{CGAC})_{17}$ & $264-320$ & 7 & $\begin{array}{l}0.4000 \\
0.5359\end{array}$ & JF692802 \\
\hline BH02 & $\begin{array}{l}\text { F: CAAATACTCTATATGTTCTGAGGG } \\
\text { R: TAAGAAATGGTCAGTGCTGGT }\end{array}$ & 50 & $(\mathrm{TC})_{32}$ & $257-259$ & 2 & $\begin{array}{l}0.2222 \\
0.3660\end{array}$ & JF692832 \\
\hline BH021 & $\begin{array}{l}\text { F: GAATGTCTGACTCATTTGTGC } \\
\text { R: AACTGACAGTCTTGTTTGACG }\end{array}$ & 48 & $(\mathrm{GTT})_{4}$ & $183-261$ & 9 & $\begin{array}{l}0.6923 \\
0.8738\end{array}$ & JF692833 \\
\hline BH05 & $\begin{array}{l}\text { F: CAACCTATTAACTCGTTATGGC } \\
\text { R: ATCCTCTACTCGACAGTCTCC }\end{array}$ & 62 & $(\mathrm{TG})_{10}$ & $132-180$ & 10 & $\begin{array}{l}0.7083 \\
0.8821\end{array}$ & JF692834 \\
\hline BJ43 & $\begin{array}{l}\text { F: TGTTCCTCGTAGTTAATAGCC } \\
\text { R: CAGCAACCTTTTCCTGTGTAT }\end{array}$ & 56 & $(\mathrm{AC})_{12}$ & $181-235$ & 11 & $\begin{array}{l}0.6087 \\
0.8870\end{array}$ & JF692803 \\
\hline
\end{tabular}

Continued on next page 
Table 1. Continued.

\begin{tabular}{|c|c|c|c|c|c|c|c|}
\hline Locus & Primer sequence $\left(5^{\prime}-3^{\prime}\right)$ & $T_{\mathrm{a}}\left({ }^{\circ} \mathrm{C}\right)$ & Repeat motifs & Range & $N_{\mathrm{A}}$ & $H_{\mathrm{O}} / H_{\mathrm{E}}$ & Accession No. \\
\hline \multirow[t]{2}{*}{ FS01 } & F: CACGTTTTGTTGACCTCACC & 48 & $(\mathrm{TG})_{33}$ & 192-194 & 2 & 0.5000 & JF692804 \\
\hline & R: TTTATCCCATCACACGAAGC & & & & & 0.5303 & \\
\hline \multirow[t]{2}{*}{ FS07 } & F: ATGGCAGTTTGGATCGAGTG & 52 & $(\mathrm{TG})_{29}$ & $311-323$ & 2 & 0.3529 & JF692805 \\
\hline & R: CGCAGCAACATCAAAAGTTC & & & & & 0.2995 & \\
\hline \multirow{2}{*}{ FS08 } & F: TACCTCATGGACAAGATTCG & 48 & $(\mathrm{GT})_{26}$ & $221-249$ & 7 & 0.6364 & JF692806 \\
\hline & R: ACACCAGTGTGTTCTCTCAAG & & & & & 0.6883 & \\
\hline \multirow[t]{2}{*}{ HC506 } & F: AATAGAACGACTGAACCAAGG & 50 & $(\mathrm{GA})_{25}(\mathrm{GT})_{14}$ & $157-171$ & 9 & 0.4783 & JF692784 \\
\hline & R: GAAAGTATCAAAAGTAGCACACG & & & & & $0.3961^{*}$ & \\
\hline \multirow[t]{2}{*}{ HD303 } & F: CATTTGGCTCGACTGACGAC & 62 & $(\mathrm{TG})_{12}$ & $199-243$ & 8 & 0.2381 & JF692863 \\
\hline & R: TAGGTCAAGGGTCAATAAAGG & & & & & $0.7422^{*}$ & \\
\hline \multirow[t]{2}{*}{ HD307 } & F: TGCTTCTTTGCGAGTTCCTG & 62 & $(\mathrm{CACG})_{4}(\mathrm{CA})_{15}(\mathrm{TACA})_{14}$ & $277-295$ & 4 & 0.6250 & JF692866 \\
\hline & R: GGATGAGATGTAGGGACCAAT & & & & & $0.7250^{*}$ & \\
\hline \multirow[t]{2}{*}{ HD308 } & F: GAGATACCGTGTTTACAAGCC & 45 & $(\mathrm{CA})_{9} \mathrm{CG}(\mathrm{CA})_{9}$ & $211-257$ & 9 & 1.0000 & JF692867 \\
\hline & R: GTCAGGACACCTATATGTTTCG & & & & & 0.8992 & \\
\hline \multirow[t]{2}{*}{ HD406 } & F: CTGACATGGATGTCCACCAG & 62 & $(\mathrm{GT})_{25}$ & $229-263$ & 11 & 0.3333 & JF692870 \\
\hline & R: ATCATGTTTACAAGCGAGGC & & & & & $0.8483^{*}$ & \\
\hline \multirow[t]{2}{*}{ HD407 } & F: GTTCCATTACCGTCCATTGC & 62 & $(\mathrm{TG})_{8}$ & $198-222$ & 7 & 0.7500 & JF692871 \\
\hline & R: GTTGGCACGTTGGAGGTTAC & & & & & $0.8271^{*}$ & \\
\hline \multirow[t]{2}{*}{ HD409 } & F: ATTCTGGTTGAGTTGGTTCG & 62 & $(\mathrm{CA})_{12} \ldots(\mathrm{CA})_{9}$ & $172-298$ & 9 & 0.6667 & JF692872 \\
\hline & R: AGATTGGAGTCTACGGTATGG & & & & & 0.8785 & \\
\hline \multirow[t]{2}{*}{ HD411 } & F: CAGAGGCTCGGTAAGTAATG & 62 & $(\mathrm{TG})_{7} \ldots(\mathrm{GT})_{9}$ & $236-252$ & 4 & 0.3750 & JF692873 \\
\hline & R: TCTATCTATGCAGTCTAGTGGC & & & & & $0.7145^{*}$ & \\
\hline \multirow[t]{2}{*}{ HD417 } & F: CACTTCAAAGAACAAACGACAG & 50 & $(\mathrm{TC})_{12}$ & $238-244$ & 3 & 0.3128 & JF692876 \\
\hline & R: CATGAGTTGTTCGTCCTTGA & & & & & 0.3943 & \\
\hline \multirow[t]{2}{*}{ HD419 } & F: CACCCAGCAAGTTTGGACATC & 58 & $(\mathrm{CA})_{9} \mathrm{CG}(\mathrm{CA})_{9}$ & $118-128$ & 3 & 0.5000 & JF692877 \\
\hline & R: GCACACGCCCGTATGTTTC & & & & & 0.4140 & \\
\hline \multirow[t]{2}{*}{ HD420 } & F: ACATGATCTTTCACCACTGCC & 62 & $(\mathrm{AC})_{14}$ & $166-170$ & 3 & 0.8235 & JF692878 \\
\hline & R: TCCTTGAATCTTTGTGATGCTG & & & & & $0.6471^{*}$ & \\
\hline HD422 & F: CTCACTCGGCTTTACTTCCT & 45 & $(\mathrm{TC})_{22}$ & $141-151$ & 4 & 0.5500 & JF692880 \\
\hline & R: CAAACATACAAGTCCATGTCC & & & & & 0.4487 & \\
\hline HD602 & F: CTTCTACTCAATGTGGTAATCC & 62 & $(\mathrm{CA})_{19}$ & $240-254$ & 5 & 0.5101 & JF692881 \\
\hline & R: CTGTTCATGTTATCTTGGGC & & & & & $0.8094^{*}$ & \\
\hline HD604 & F: AACATGGCAGAATTCAACGAC & 45 & $(\mathrm{TG})_{24}$ & $202-218$ & 6 & 0.2857 & JF692882 \\
\hline & R: GCACACCACTTACTCAAAACAC & & & & & $0.6876^{*}$ & \\
\hline HD608 & F: TGTGACAATCAACAACAACC & 55 & $(\mathrm{CA})_{17} \mathrm{CGCAT}(\mathrm{AC})_{10}$ & $154-172$ & 8 & 0.3529 & JF692883 \\
\hline & R: TTCATACTAATGGGAAAGGG & & & & & $0.8217^{*}$ & \\
\hline HS16 & F: CTAACCGCTTCACTAGGCTTTG & 62 & $(\mathrm{GA})_{8} \mathrm{TGG}(\mathrm{GA})_{3}$ & $265-307$ & 4 & 0.4167 & JF692785 \\
\hline & R: CGTCATGTTTCATTGTCATCG & & & & & $0.5955^{*}$ & \\
\hline HS38 & F: AGGGATTGCCTAATCATTATG & 60 & $(\mathrm{GT})_{2} \mathrm{ATGTCT}(\mathrm{GT})_{5}$ & $214-248$ & 7 & 0.0909 & JF692786 \\
\hline & R: CACTTGATCTGAGTATTCTGC & & & & & 0.8223 & \\
\hline PS205 & F: GACTACTATAGCAGTGACCTCG & 56 & $(\mathrm{CA})_{12}$ & $144-160$ & 3 & 0.1818 & JF692836 \\
\hline & R: GTTTACATCTATCCATGTGATTG & & & & & 0.5032 & \\
\hline PS245 & F: ACTTAACCACACACCAGAAATG & 52 & $(\mathrm{GT})_{11} \mathrm{AC}(\mathrm{GT})_{7}$ & $158-206$ & 7 & 0.7391 & JF692838 \\
\hline & R: ACCAATCACACTGGCACAAG & & & & & $0.6715^{*}$ & \\
\hline PS440 & F: AAATACAACCCTGGGAAGCC & 62 & $(\mathrm{GA})_{19}$ & $123-207$ & 4 & 0.6667 & JF692839 \\
\hline & R: CACCCGAAGACGAAATGAAC & & & & & $0.7316^{*}$ & \\
\hline PS445 & F: CACTTATTTTCTCACCAGACACG & 45 & $(\mathrm{AG})_{10} \mathrm{ATG}(\mathrm{GA})_{24}$ & $229-273$ & 5 & 0.6364 & JF692841 \\
\hline & R: CGCAACTCAACAAGTTGTGC & & & & & 0.7219 & \\
\hline PS457 & F: GGGGATGAGATGTAGGGACC & 54 & $(\mathrm{TATG})_{15}(\mathrm{TG})_{14}$ & $324-413$ & 6 & 0.4118 & JF692842 \\
\hline & R: ACACGAGCTTGCATACCTGC & & & & & $0.7241^{*}$ & \\
\hline PS462 & F: TCAGTTCCTTATATCTCGTTCT & 45 & $(\mathrm{TC})_{22}$ & $165-189$ & 4 & 0.8000 & JF692844 \\
\hline & R: AATTCATTTACGGGCAGGTC & & & & & $0.7000^{*}$ & \\
\hline PS470 & F: CAGCCATCTCATTTTGTTCTAC & 62 & $(\mathrm{TG})_{8} \ldots(\mathrm{GT})_{7}$ & $259-299$ & 5 & 0.2914 & JF692845 \\
\hline & R: CAGATAATACCACTTTACACCG & & & & & $0.4848^{*}$ & \\
\hline PS474 & F: CAATCAGATGCGATTTAGAGAC & 62 & $(\mathrm{CT})_{15} \ldots(\mathrm{CT})_{10}$ & $267-348$ & 7 & 0.3913 & JF692846 \\
\hline & R: ATACCCTTCCTATCCTATCCAG & & & & & $0.6947^{*}$ & \\
\hline QS06 & F: AAATACAACCCTGGGAAGCC & 62 & $(\mathrm{GA})_{22}$ & $160-230$ & 4 & 0.6667 & JF692807 \\
\hline & R: AGCCGTAGACGAATTGAACC & & & & & 0.5151 & \\
\hline WS68 & F: GTCCGAATTAGAACATAGAGAC & 48 & $(\mathrm{AG})_{29}$ & $189-243$ & 6 & 0.2667 & JF692835 \\
\hline & R: AGCCCAAATTGAACGAATAG & & & & & $0.7770^{*}$ & \\
\hline XS03 & F: ATACAACACCCTGACATAGCG & 62 & $(\mathrm{GT})_{10} \ldots(\mathrm{TG})_{10}$ & $271-303$ & 10 & 0.5217 & JF692819 \\
\hline & R: TGAGATAGAGCGTACCCAAG & & & & & 0.8386 & \\
\hline
\end{tabular}

Continued on next page 


\begin{tabular}{|c|c|c|c|c|c|c|c|}
\hline Locus & Primer sequence $\left(5^{\prime}-3^{\prime}\right)$ & $T_{\mathrm{a}}\left({ }^{\circ} \mathrm{C}\right)$ & Repeat motifs & Range & $N_{\mathrm{A}}$ & $H_{\mathrm{O}} / H_{\mathrm{E}}$ & Accession No. \\
\hline XS05 & $\begin{array}{l}\text { F: TGAGGAGGAAGACGATCTATC } \\
\text { R: ATTCGGACTATATCACCCCAT }\end{array}$ & 56 & $(\mathrm{CA})_{12}$ & $131-185$ & 9 & $\begin{array}{l}0.7778 \\
0.8746\end{array}$ & JF692820 \\
\hline XS06 & $\begin{array}{l}\text { F: GTTCATCTACTCACTATGGGC } \\
\text { R: ATACTGTCACATCAAGCACG }\end{array}$ & 62 & $(\mathrm{CA})_{13} \mathrm{~T}(\mathrm{CA})_{10}$ & $184-256$ & 10 & $\begin{array}{l}0.5714 \\
0.9101\end{array}$ & JF692808 \\
\hline XS19 & $\begin{array}{l}\text { F: ATGGAGCAACTGTGTGTCAAG } \\
\text { R: GCCAAAGGCAGGTATTGTAAC }\end{array}$ & 62 & $(\mathrm{CA})_{14}$ & $147-179$ & 8 & $\begin{array}{l}0.6250 \\
0.7961\end{array}$ & JF692812 \\
\hline XS20 & $\begin{array}{l}\text { F: GATAGCACAAGCCAAGCGTC } \\
\text { R: GCCACAGAGAGGAGTTATTCAG }\end{array}$ & 62 & $(\mathrm{CA})_{28}$ & $122-238$ & 14 & $\begin{array}{l}0.6250 \\
0.9415\end{array}$ & JF692813 \\
\hline $\mathrm{XS} 25$ & $\begin{array}{l}\text { F: CCCATGAACATGCTAACAGAAG } \\
\text { R: ACCCAAGCAAGTGTCACAGAC }\end{array}$ & 62 & $(\mathrm{GT})_{8} \mathrm{~A}(\mathrm{TG})_{11}$ & $244-278$ & 13 & $\begin{array}{l}0.6818 \\
0.8890\end{array}$ & JF692814 \\
\hline XS32 & $\begin{array}{l}\text { F: TATTTGTGCAGGATCGAGGC } \\
\text { R: ATAGGTGGAGGAAGCATTGG }\end{array}$ & 56 & $(\mathrm{GT})_{15}$ & $121-177$ & 8 & $\begin{array}{l}0.7692 \\
0.8800\end{array}$ & JF692816 \\
\hline XS33 & $\begin{array}{l}\text { F: CGTGTGCGTGTAAATGTTTG } \\
\text { R: GAGTGTGTTGTGGTCTCAGTC }\end{array}$ & 60 & $(\mathrm{CT})_{25}$ & $186-248$ & 14 & $\begin{array}{l}0.7143 \\
0.9471\end{array}$ & JF692817 \\
\hline XS34 & $\begin{array}{l}\text { F: GGCTATTGTGGCTGTGAAGTC } \\
\text { R: AGTGGAGTCATTGAAAGGCG }\end{array}$ & 60 & $(\mathrm{TG})_{59}$ & $251-287$ & 5 & $\begin{array}{l}0.6250 \\
0.6750\end{array}$ & JF692818 \\
\hline
\end{tabular}

$T_{\mathrm{a}}=$ annealing temperature; Range $=$ size range of alleles; $N_{\mathrm{A}}=$ number of alleles; $H_{\mathrm{O}}=$ observed heterozygosity; $H_{\mathrm{E}}=$ expected heterozygosity. *Indicates significant deviation from HWE after Bonferroni's correction $(\mathrm{P}<0.01)$.

\section{ACKNOWLEDGMENTS}

Financial support for this study was provided by the National Key Technology R\&D Program of China (\#2011BAD13B05 and \#2011BAD13B06), and the National High Technology Research and Development Program of China (\#2012AA10A412).

\section{REFERENCES}

Chen JX (1990). Brief Introduction to Mariculture of Five Selected Species in China. In: UNDP/FAO Regional Seafarming Development and Demonstration Project, National Inland Fisheries Institute. Kasetsart University Campus, Bankok, 16.

Chen L and Li Q (2007). Identification and characterization of microsatellite markers derived from expressed sequence tags (ESTs) of the sea cucumber Stichopus japonicus. Mol. Ecol. Notes 7: 1057-1059.

Fischer D and Bachmann K (1998). Microsatellite enrichment in organisms with large genomes (Allium cepa L.). Biotechniques 24: 796-800, 802.

Kanno M, Li Q and Kijima A (2005). Isolation and characterization of twenty microsatellite loci in Japanese sea cucumber (Stichopus japonicus). Mar. Biotechnol. 7: 179-183.

Peng W, Bao Z, Du H, Dong Y, et al. (2009). Development and characterization of 38 novel EST-SSRs for the sea cucumber Apostichopus japonicus. Conserv. Genet. Resour. 1: 447-450.

Rice WR (1989). Analyzing tables of statistical tests. Evolution 43: 223-225.

Schneider S, Roessli D and Excoffier L (2000). ARLEQUIN: A Software for Population Genetics Data Analysis, Version 2.0. Genetics and Biometry Laboratory, Department of Anthropology. University of Geneva, Geneva.

Yeh FC and Boyle TJB (1997). Population genetic analysis of co-dominant and dominant markers and quantitative traits. Belg. J. Bot. 129: 157.

Zhan A, Bao Z, Lu W, Hu X, et al. (2007). Development and characterization of 45 novel microsatellite markers for sea cucumber (Apostichopus japonicus). Mol. Ecol. Resour. 7: 1345-1348. 